\title{
Mapeamento Sistemático da Literatura acadêmico- cientifica sobre Análise de Redes Sociais aplicada em E-Learning
}

\author{
Systematic Mapping of the academic and scientific literature on Social Network \\ Analysis applied in E-Learning
}

Jarbele Cássia da Silva

Centro de Informática

Universidade Federal da Paraíba

UFPB - João Pessoa - PB - Brasil

jarbele.cassia@gmail.com

\author{
Alisson Vasconcelos de Brito \\ Centro de Informática \\ Universidade Federal da Paraíba \\ UFPB - João Pessoa - PB - Brasil \\ alisson@ci.ufpb.br
}

\author{
Francisco P. A. de Medeiros \\ Instituto Federal de Educação, \\ Ciência e Tecnologia da Paraíba \\ IFPB - João Pessoa - PB - Brasil \\ petronio@ifpb.edu.br
}

Resumo Este trabalho apresenta os resultados de um mapeamento sistemático da literatura, na língua inglesa, acerca dos artigos científicos que versam sobre a Análise de Redes Sociais (SNA) aplicadas em modelos de ensino E-Learning, com o intuito de identificar as vertentes dadas a ELearning quando a SNA é inserida neste contexto, bem como, explorar as possibilidades de estudo sobre esta temática. Esta pesquisa permitiu perceber que poucos trabalhos evidenciam o uso de métricas de SNA aplicadas em E-learning para examinar seus experimentos e que o principal foco das pesquisas cientificas que versam sobre esta temática tem sido medir o índice de isolamento ou engajamento dos alunos por meio da análise da interação existente em fóruns de discussão online. Os resultados desse trabalho geram a fundamentação teórica para, em um passo seguinte, a investigação sobre a interação que ocorre nos fóruns de discussão, de modo a perceber se este tipo de interação online pode impactar no desempenho do aluno em cursos à distância, cuja investigação será realizada através das métricas de Análise de Redes Sociais.

Palavras-Chave: Análise de Redes Sociais, E-Learning, Mapeamento Sistemático

\begin{abstract}
This paper presents the results of a bibliographic study of literature, in English, about the scientific articles that deal with the Social Network Analysis (SNA) applied in teaching models Elearning, with the aim of identifying aspects given E-Learning when SNA is inserted in this context, as well as exploring the possibilities of study on this topic. This research allowed us to realize that few studies show the use of SNA metrics applied in E-learning to examine their experiments, and that the main focus of scientific research that deal with this issue has been measuring the insulation index or student engagement through the analysis of existing interaction in online discussion forums. The findings generate the theoretical foundation for, in a next step, research on the interaction that occurs in the discussion forums, in order to understand if this type of online interaction can impact the performance of the student in distance courses, whose research will be conducted through the metrics of Social Network Analysis.
\end{abstract}

Keywords: Social Network Analysis, E-Learning, Systematic Mapping 


\section{Introdução}

Para [1] as redes sociais referem-se a um conjunto de pessoas, organizações ou outras entidades conectadas por relacionamentos sociais, motivados pela amizade, por relações de trabalho ou compartilhamento de informações, que por meio dessas ligações, vão construindo e reconstruindo a estrutura social.

Hoje, a utilização das redes sociais mediadas pela Internet trouxe este tipo de estrutura social para um novo contexto, com uma abrangência social, econômica e geográfica antes impraticável. Temos então as Redes Sociais Online que além de proporcionarem um ambiente propício ao compartilhamento de informações, assumem principalmente um caráter interativo dentro deste contexto.

Diante desta nova percepção foi notado que o agrupamento em redes sociais online também é construído no âmbito educativo, sobretudo em modelos de ensino não presenciais suportados por tecnologia, ou seja, em modelos de ensino/aprendizagem E-Learning. Neste formato de ensino, à distância, as pessoas não estão apenas reunidas em torno de um tema. Há uma proposta a cumprir, situações didáticas e alguém do grupo que provoca e estimula a participação dos demais. Essas características distinguiriam as chamadas redes e comunidades virtuais de aprendizagem dos demais agrupamentos na Internet [2].

Assim sendo, surge a necessidade de se realizar uma análise mais específica dos relacionamentos, atividades e informações geradas por meio do monitoramento das interações destes agrupamentos. A Análise de Redes Sociais (SNA) estuda como os comportamentos ou as opiniões dos indivíduos dependem das estruturas nas quais eles se inserem, onde a unidade de análise não são os atributos individuais (classe, sexo, idade, gênero), mas o conjunto de relações que os indivíduos estabelecem através das suas interações uns com os outros [5].

Visando complementar as pesquisas que relacionam SNA e E-Learning, a proposta deste trabalho é levantar dados de forma sistemática para fundamentar o desenvolvimento de estudos mais específicos acerca da utilização de Análise de Redes Sociais na Educação, especialmente em E-Learning, de modo, a contribuir com as abordagens até então adotadas para a análise das interações em modelos de ensino à distância.

Desta forma, o trabalho organiza-se em seções que descrevem as ideias e os resultados do estudo. A seção 2 apresenta uma breve contextualização sobre Análise de Redes Sociais e E-Learning. A seção 3 descreve os procedimentos metodológicos aplicados a este estudo. A seção 4 traz os resultados e a análise das informações obtidas com a pesquisa. E, a seção 5 , é dedicada as considerações finais da pesquisa e proposta de trabalhos futuros.

\section{Análise de Redes Sociais e E-Learning}

O modelo de ensino não presencial suportado por tecnologia tem se tornado uma prática cada vez mais utilizada em educação, principalmente no que se refere ao modelo de Educação a Distância. Para isto, diversas ferramentas, síncronas e assíncronas estão presentes em ambientes de gestão de aprendizagem que apoiam a colaboração entre as pessoas, tais como fóruns, chats, e-mails, e mais recentemente as redes sociais online [7], conectadas ou não aos Ambientes Virtuais de Aprendizagem (AVA).

Esta recente modalidade de ensino tem proporcionado um diferencial no acesso a educação ao flexibilizar as restrições de tempo e espaço [4], ao proporcionar novas formas de interação e colaboração, dentre outros fatores. Nesse contexto passa a existir uma preocupação sobre como acompanhara dinâmica dos processos (níveis de aprendizagem, participação, evasão etc.) que envolvem os aprendizes neste ambiente. Entretanto, as ferramentas disponíveis no AVA, por si só, não disponibilizam estratégias para realização de uma análise precisa de tais fatores. Essa só pode ser efetivada através do uso de métricas específicas de análise e avaliação.

Assim sendo, podemos entender que as ferramentas de um AVA também encorporam as Redes Sociais Online, uma vez que são ambientes que proporcionam um espaço bastante propício para o compartilhamento de ideias, informações e valores, assumindo um caráter interativo [1].

A Análise de Redes Sociais estabelece um paradigma na pesquisa sobre a estrutura social. Através dela é possível compreender e acompanhar de forma mais eficaz a disseminação de informações e a interação entre as pessoas que compõem a rede [7], por meio do uso de métricas de SNA que possibilitam a identificação de relacionamentos, bem como, sua interpretação.

O objetivo geral desta pesquisa é investigar e reunir de forma sistemática os trabalhos científicos já publicados que versam sobre SNA em sistemas E-learning, oferecendo respostas para dois momentos distintos desse processo: o primeiro, relacionado a identificação das vertentes dadas a E-Learning quando a SNA é inserida neste contexto e o segundo, faz referencia as possibilidades de estudo sobre esta temática.

\section{Metodologia}

A pesquisa bibliográfica é uma importante etapa no processo de produção científica, pois fornece uma visão geral da área que se pretende investigar, possibilitando um amadurecimento da pesquisa em questão. Nesse sentido, existem diferentes métodos que sistematizam o trabalho de obtenção de dados e apresentação dos resultados desse tipo 
de pesquisa, entre eles, destaca-se o Mapeamento Sistemático da Literatura (SLM, Systematic Literature Mapping).

De acordo com Kitchenham [3], um Mapeamento Sistemático é realizado para identificar, avaliar e interpretar os estudos que estejam disponíveis e que sejam relevantes a uma determinada questão de pesquisa. Através dele é possível identificar se uma área possui estudos primários suficientes para realização de uma Revisão Sistemática de Literatura ou ainda se a área em questão possui subtemas pouco explorados em estudos primários.

O Mapeamento Sistemático descrito neste documento objetiva identificar, na literatura científica de conferências e revistas, na língua inglesa, quais são os trabalhos que relacionam o estudo da Análise de Redes Sociais e Elearning já publicados. Para isto, esta pesquisa segue as diretrizes classificadas por [3]. Assim sendo, ela pode ser realizada com dois objetivos distintos: fornecer um conjunto relevante de trabalhos relacionados para fundamentar novas pesquisas sobre SNA e E-Learning ou identificar lacunas existentes nesta área de pesquisa. Esta seção apresenta as principais decisões tomadas durante o estudo: o processo de busca, a seleção e a extração das informações dos estudos primários apurados, embasados na metodologia supracitada.

\subsection{Questões de pesquisa}

Com o objetivo de estudar o estado atual do conhecimento acerca dos benefícios e limitações da Análise de Redes Sociais no contexto de E-learning, assim como a implicação dos estudos existentes na área para a comunidade acadêmica e científica, foi definida como questão central de pesquisa a seguinte pergunta: Qual o panorama atual de pesquisa das publicações científicas, em língua inglesa, sobre o uso de SNA no contexto de E-learning? Como consequência desse questionamento foram definidas as seguintes questões específicas de pesquisa:

Q1. Qual o foco dado a E-learning quando a SNA é inserida neste contexto?

Q2. Quais as possibilidades de estudo sobre SNA em $E$ learning?

\subsection{Estratégia e processo de busca}

Neste mapeamento foi utilizada uma string de busca simples, com as palavras-chaves "Social Network Analysis" AND E-Learning, em diferentes ferramentas de busca, a citar: ACM Digital Library (http://www.acm.org/), Elsevier Scopus (www.elsevier.com/online-tools/scopus), IEEE Digital Library (www.ieee.org.br/), Springer Link (link.springer.com/) e Science Direct (www.sciencedirect.com/). Esta busca identificou exatamente 187 artigos científicos que apresentavam em seus títulos, resumos e/ou palavras-chaves os vocábulos mencio- nados. A partir dessa identificação foi possível iniciar uma análise mais aprofundada dos trabalhos relacionados à temática em foco e gerar os resultados apresentados nas seções posteriores.

\subsection{Critérios de inclusão e exclusão}

Para a inclusão de um trabalho na pesquisa foi determinada sua relevância em relação às questões de investigação, determinada pela análise do título, palavras-chave e resumo. Especificamente, dois critérios de inclusão foram definidos:

- Estudos com foco no contexto de SNA e $E$ learning;

- Apresentação de resultados completos (com experimentos e análise dos resultados).

Depois de aplicados os critérios de inclusão foram aplicados os critérios de exclusão. A partir da análise do título, palavra-chave e resumo, foram excluídos os estudos que se enquadraram em algum dos critérios a seguir:

(i) Estudos que se encontram repetidos ou que foram publicados em mais de uma fonte de busca.

(ii) Estudos sem acesso disponível para visualização e/ou download do trabalho.

(iii) Estudos incompletos (em termos de conteúdo, texto ou resultados).

(iv) Estudos com conteúdo irrelevante em relação aos domínios de pesquisa.

A aplicação de apenas um critério de inclusão ou exclusão determinou se o artigo deveria ser incluído ou excluído, respectivamente.

\subsection{Extração e síntese de dados}

Neste âmbito, foram extraídos alguns dados gerais, como: título, autor (es), ano de publicação, entre outras informações peculiares de cada trabalho. Além disso, foram extraídos também trechos de alguns artigos que responderam as perguntas de pesquisa que nortearam este Mapeamento Sistemático. Os resultados são expostos e discutidos na seção que segue.

\section{Resultados e Análises}

Esta seção apresenta os resultados encontrados no Mapeamento Sistemático realizado a partir dos trabalhos primários selecionados, bem como a descrição das análises efetuadas a partir dos mesmos.

\subsection{Informações gerais sobre os trabalhos primários}


Foram retornados por todas as fontes de busca supracitadas 187 (cento e oitenta e sete) trabalhos primários, sendo extraídos neste sentido, os seguintes dados gerais: título, autores, evento, ano de publicação, país de origem da instituição de pesquisa e fonte de publicação. Em seguida, uma melhor análise dos estudos primários foi realizada identificando como relevantes apenas àqueles que se enquadravam nos critérios de inclusão e exclusão expostos na seção 3.3, permanecendo na pesquisa 34 (trinta e quatro) artigos científicos. Após esta etapa, foi feita a leitura completa dos artigos por cada pesquisador, buscando identificar as especificidades de cada trabalho (essas informações são descritas na subseção 4.2).

A Tabela 1 apresenta de forma sucinta a evolução do processo de seleção dos trabalhos primários. As ferramentas de busca estão listadas, na primeira coluna. A segunda coluna mostra o número de artigos científicos, em ordem decrescente, de acordo com a quantidade de estudos retornados na primeira etapa do processo do Mapeamento Sistemático. Na terceira coluna é exposta a quantidade de artigos selecionados que continham os vocábulos "Social Network Analysis" e "E-learning” em seu título, resumo e/ou palavras-chaves. As colunas finais da tabela apresentam o número de artigos selecionados, segundo os critérios de exclusão e inclusão.

Ao analisar as fontes de busca separadamente, podemos notar que a Scopus e a Springer Link são as bibliotecas que possuem um número maior de artigos publicados relacionados com a string de busca utilizada neste trabalho.

É válido mencionar que, embora o Scopus apresente um número expressivo de trabalhos retornados, ao final da seleção apenas 1 (um) trabalho primário foi incluído. Já, a IEEE Xplorer foi a fonte que retornou menos estudos, quando comparado às demais fontes de busca utilizadas.

No total foram excluídos na segunda fase da seleção 90 (noventa) trabalhos primários, sendo que: 11 (onze) apareceram repetidos em mais de uma ferramenta de busca, 53 (cinquenta e três) não estavam disponíveis para visualização e/ou download, 5 (cinco) continham resultados incompletos e 21 (vinte e um) apresentavam conteúdo irrelevante à pesquisa. Ao final foram incluídos 34 (trinta e quatro) trabalhos primários.

\begin{tabular}{|c|c|c|c|c|c|c|c|}
\hline \multirow[b]{4}{*}{ Ferramentas de busca } & leção de $t$ & \multirow{4}{*}{$\begin{array}{l}1^{\text {a }} \text { Seleção } \\
\text { Trabalhos } \\
\text { potencialmente } \\
\text { relevantes }\end{array}$} & & & & & \\
\hline & \multirow[b]{3}{*}{$\begin{array}{l}\text { Trabalhos } \\
\text { retornados }\end{array}$} & & \multicolumn{5}{|c|}{$2^{\text {a }}$ Seleção } \\
\hline & & & \multicolumn{4}{|c|}{ Excluídos } & \multirow{2}{*}{$\begin{array}{l}\text { Incluídos } \\
\text { Trabalhos } \\
\text { primários }\end{array}$} \\
\hline & & & 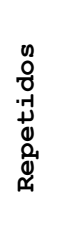 & 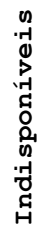 & 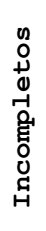 & 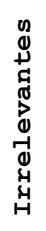 & \\
\hline Scopus & 46 & 26 & - & 24 & - & 01 & 01 \\
\hline Springer Link & 44 & 26 & - & 14 & - & 07 & 05 \\
\hline Science Direct da Elsevier & 37 & 28 & 11 & 01 & 01 & 02 & 13 \\
\hline ACM Digital Library & 35 & 25 & - & 01 & 04 & 10 & 10 \\
\hline IEEE Xplorer & 25 & 19 & - & 13 & - & 01 & 05 \\
\hline TOTAL & 187 & 124 & 11 & 53 & 05 & 21 & 34 \\
\hline
\end{tabular}

Tabela 1: Evolução do processo de seleção dos trabalhos primários

Apesar da busca não ter sido limitada a um período de tempo, todos os trabalhos primários incluídos compreendem os anos de 2004 a 2014, sendo a maioria concentrada nos anos de 2012 e 2013. O gráfico da Figura 1 ilustra a concentração dos estudos por ano de publicação.

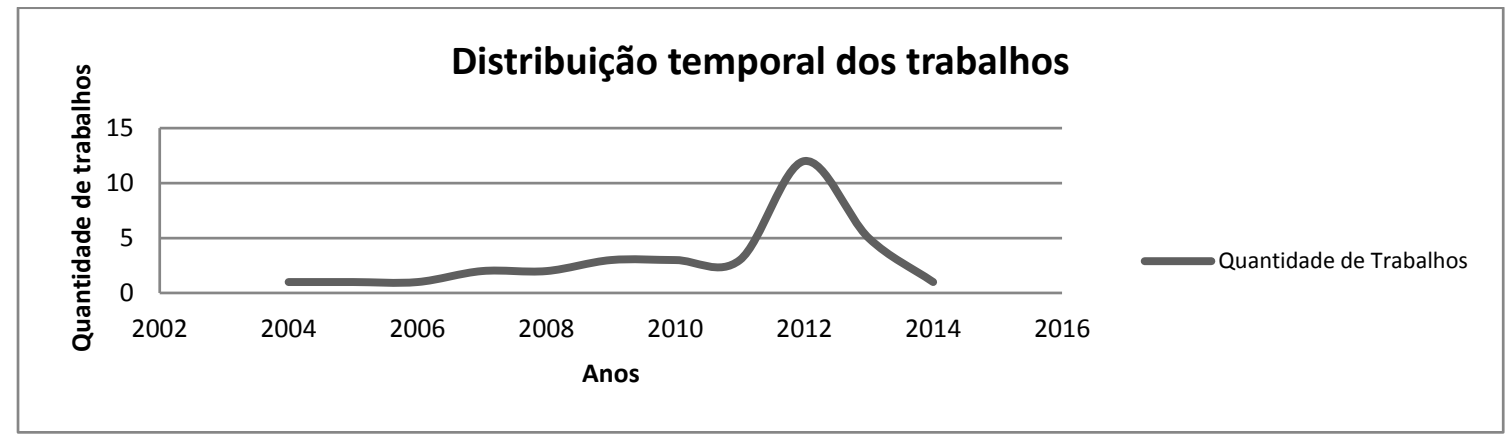

Figura 1: Distribuição dos trabalhos primários ao longo dos anos 
Na sequência foram analisados os países de origem das publicações. Os Estados Unidos lidera a lista com uma frequência de 9 (nove) artigos publicados, representando $26 \%$ do total, seguido pela Espanha com 5 (cinco) artigos, $15 \%$ da quantidade total, e pelo Canadá com 3 (três) artigos, equivalente a 9\%. Observa-se uma concentração entre esses três países, pois são responsáveis por $50 \%$ das publicações sobre o tema enquanto que os outros $50 \%$ restantes estão distribuídos entre os demais países.

A Figura 2 apresenta os 16 (dezesseis) países com a quantidade de publicações respectivas.

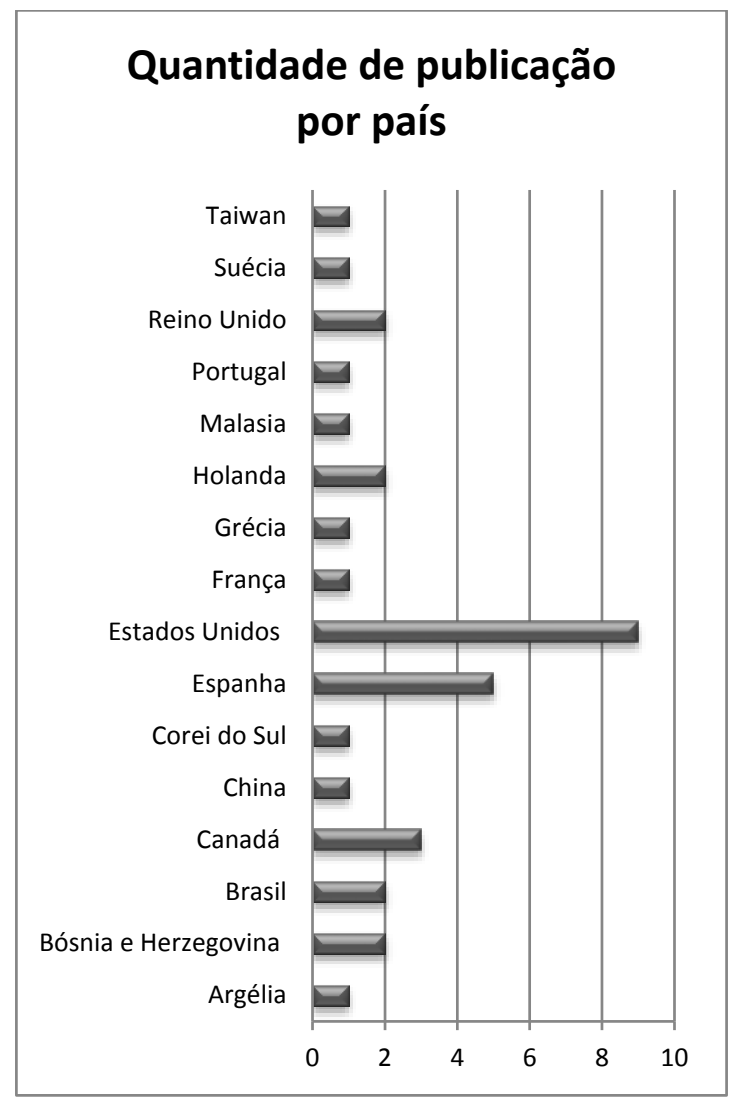

Figura 2: Países com maior frequência de publicações

Foram analisadas também as principais referências de publicação (conferências ou revistas) acerca desta temática. Percebeu-se, portanto, que $21 \%$ dos trabalhos primários foram publicados no International Journal of Computers \& Education, 12\% deles no LAK'12: Proceedings of the 2nd International Conference on Learning Analytics and Knowledge, outros 9\% no Procedia Social and Behavioral Sciences, e os 58\% restantes estão distribuídos entre as demais conferências e/ou revistas científicas (vide Figura 3).

\section{Quantidade de publicações por conferência/revista}

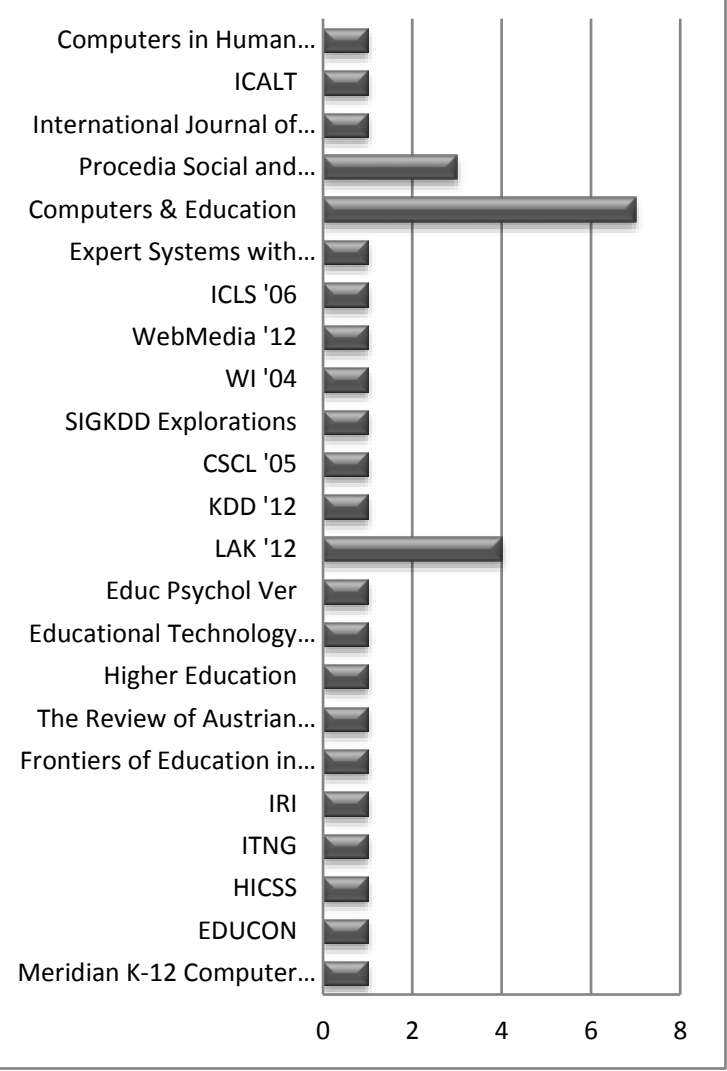

Figura 3: Quantidade de publicações por conferência/revista

\subsection{Informações peculiares sobre os traba- lhos primários}

Ao analisar os trabalhos primários individualmente, por meio da leitura completa de seu conteúdo, algumas características foram extraídas a fim de auxiliar o desenvolvimento de atividades de pesquisa posteriores. Assim, esta seção evidencia o mapeamento de informações específicas, sobre o tema abordado nesta pesquisa, encontradas a partir da análise dos trabalhos primários.

\section{A) Trabalhos primários que relacionam SNA e $E$ - learning simultaneamente}

A Análise de Redes Sociais aplicadas em E-learning estabelece um diferente paradigma de pesquisa sobre a estrutura social educacional, visto as possibilidades de analisar as interações entre os usuários pertencentes a este contexto de forma diferenciada dos métodos tradicionais até então adotados. $\mathrm{Na}$ literatura inglesa mais recente, encontram-se alguns poucos trabalhos que tratam desta temática (vide Figura 4). 
De acordo com a análise realizada foram identificados 15 (equivalente a 44\%) trabalhos primários que relacionam em suas pesquisas o estudo da SNA e E-learning. Enquanto que alguns dos estudos tratam apenas de SNA (32\% dos trabalhos) e outros apenas de E-learning (24\% dos trabalhos).

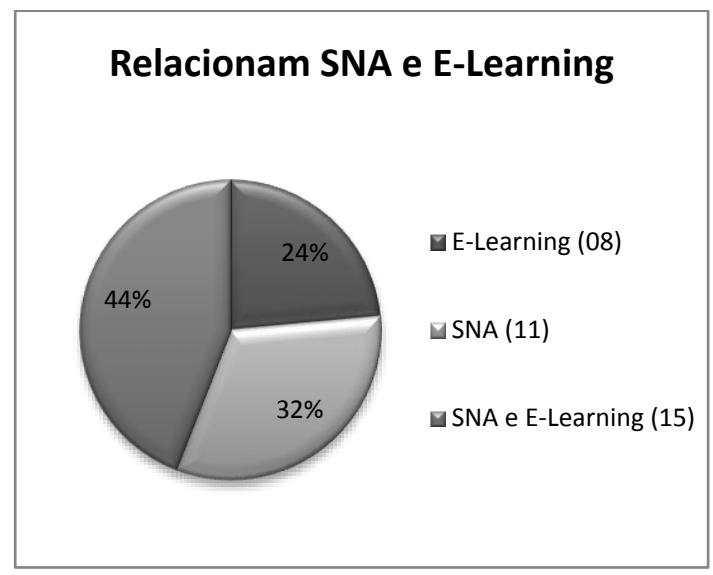

Figura 4:Trabalhos primários que relacionam SNA e E-Learning em seus estudos

\section{B) Trabalhos primários que utilizam métricas de SNA}

Em Análise de Redes Sociais existem diferentes métricas que permitem analisar e avaliar grafos, conforme a sua necessidade. Para isto, as principais métricas utilizadas são: Centrality Degree, Closeness Centrality, Betweenness Centrality, Eingevector, PageRank, Indegree, Outdegree, entre outros.

$\mathrm{Na}$ análise de trabalhos primários foi percebido que dentre as publicações que abordam o estudo de SNA, $47 \%$ das pesquisas (equivalente a 16) fazem uso de métricas de SNA para avaliar os relacionamentos existentes em suas redes. Enquanto que 3\% fazem uso de algoritmos ou outras metodologias e/ou métricas de análise. Os $50 \%$ restantes não apresentam nenhum tipo de métrica ou algoritmo de análise de grafos. Essas informações são ilustradas na Figura 5

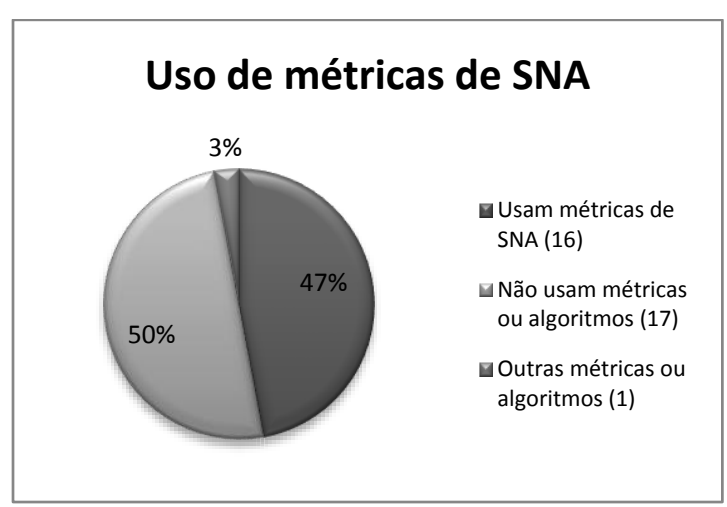

Figura 5: Trabalhos primários que utilizam métricas de SNA
Dentre os 16 trabalhos que utilizam métricas de SNA em seus experimentos, destacam-se o uso das seguintes métricas: Betweeness, Closeness, Eingenvector, Pagerank, Node Degree, Centrality Degree, Centralization Index, Density, Cohesion, Clustering e Eccentricity.

C) Trabalhos primários que realizaram suas pesquisas em AVA

A aprendizagem colaborativa se destaca como aspecto pedagógico relacionado a E-learning. É evidente que a aprendizagem colaborativa não é exclusiva do ensino a distância, mas os maiores aprofundamentos nesta área se devem a rápida expansão de cursos e faculdades nesta modalidade de ensino. Assim, os AVA surgem para atuar como salas de aula virtuais, proporcionando várias possibilidades de interações entre seus participantes [6].

Assim, outro fator avaliado a partir da análise dos trabalhos primários foi a identificação do AVA utilizado na pesquisa. Foi constatado, portanto, que $17 \%$ dos trabalhos primários utilizam o Moodle. Enquanto que $15 \%$ dos trabalhos analisados utilizam AVAs para mediação da aprendizagem, mas não mencionam o ambiente utilizado. E, em $47 \%$ dos trabalhos não há a utilização de nenhum AVA. Os demais trabalhos utilizam outros tipos de ambientes colaborativos (vide Figura 6), como: Amadeus, HyperManyMedia, CLAS, PjBL, FICTS, FIT Community Server (FITCS) e LAAM.

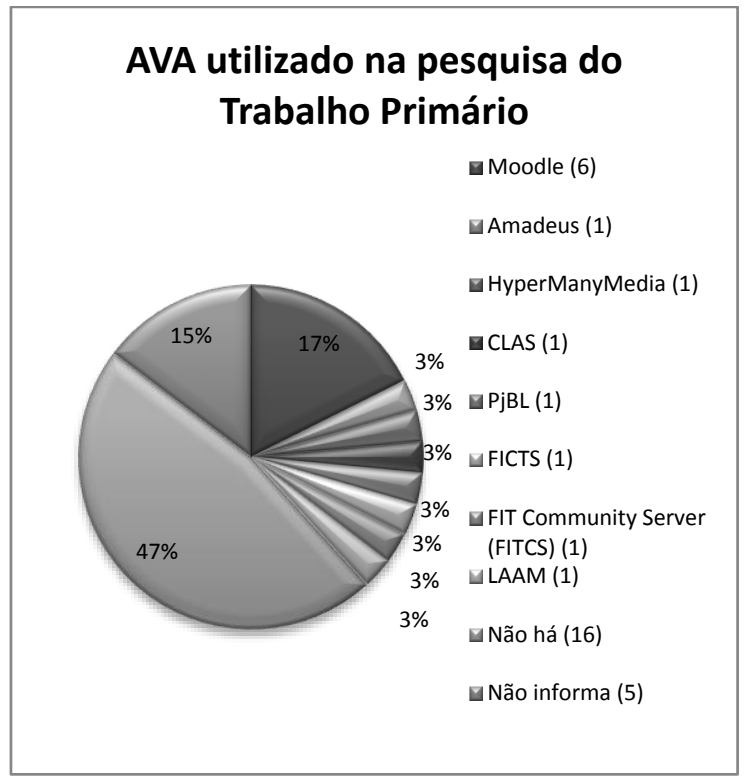

Figura 6: AVA utilizados nos trabalhos primários

\section{D) Trabalhos primários que analisam a interação em E-learning}

Analisar interações entre usuários em um contexto online não é uma tarefa trivial. Uma vez que este contexto engloba diferentes tecnologias, tais como fóruns, blogs, chats, wikis, entre outros. Nesta avaliação focamos no 
reconhecimento das abordagens usadas para análise da interação no ambiente online. Percebeu-se, portanto, que a interação nas plataformas de ensino online é mais avaliada em fóruns de discussão (32\% dos trabalhos primários), seguido da interação presencial entre os usuários ( $9 \%$ das pesquisas avaliadas), da interação através das Redes Sociais (equivalente a mais $3 \%$ ) e da análise do acesso ao conteúdo (3\% das pesquisas avaliadas). Os 53\% dos trabalhos restantes não fazem menção a análise de interação (vide Figura 7).

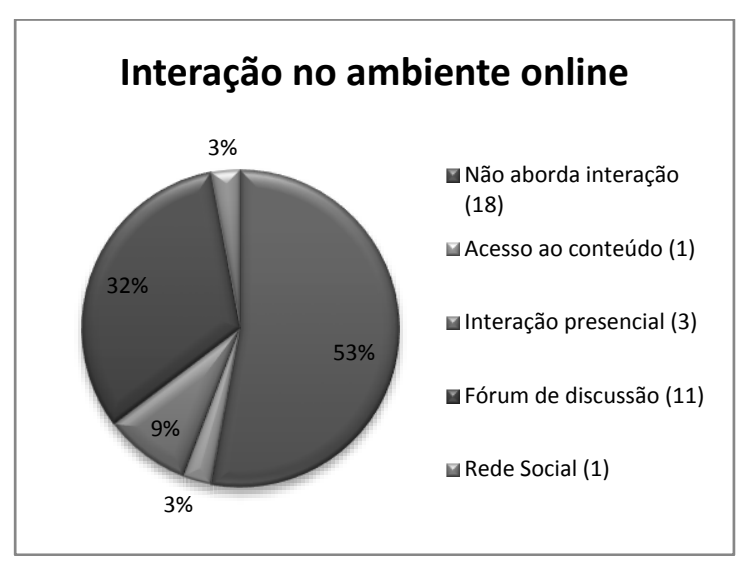

Figura 7: Foco dado a análise da interação no ambiente online

\subsection{Respostas às questões de pesquisa}

Esta seção mostra o mapeamento das evidências encontradas nos trabalhos primários como respostas aos questionamentos levantados nas duas questões propostas na seção 3.1 .

\section{Q1. Qual o foco dado a E-learning quando a SNA é inserida neste contexto?}

Essa questão visa descobrir os principais focos temáticos abordados nos trabalhos primários encontrados. O termo foco, aqui, é atribuído a fóruns de discussão, acesso ao conteúdo, interação no ambiente online, etc.

A análise realizada a partir desse questionamento identificou que o principal foco das publicações a cerca de SNA e E-learning tem sido a interação dos alunos no AVA (vide Figura 7), mais especificamente a interação que ocorre em fóruns de discussão. Isso se deve ao fato de professores e tutores utilizarem frequentemente os fóruns para discussão de conteúdos curriculares ou para estabelecer um canal eficiente de comunicação entre alunos e professores no próprio ambiente. Boa parte dos estudos analisados evidenciam essa perspectiva e buscam identificar, a partir da interação, os alunos que estão mais isolados na rede (não interagem com assiduidade) e/ou aqueles que estão mais engajados (interagem entre si com assiduidade). A SNA colabora na identificação desses indivíduos e torna-se relevante para a E-Learning porque permite compreender de modo mais preciso um dos motivos que incidem com a evasão de alunos, o isolamento no AVA [6].

Assim sendo, o passo seguinte desta pesquisa torna-se viável, visto que existem caminhos ainda pouco explorados no que se refere ao estudo da interação em fóruns de discussão em um curso à distância, segundo as métricas de Análise de Redes Sociais.

\section{Q2. Quais as possibilidades de estudo sobre SNA em E-learning?}

Nesta questão foram investigadas as possibilidades de estudo sobre SNA em E-learning. Entende-se possibilidades de estudo como desafios ainda não explorados e/ou problemáticas em aberto para pesquisas futuras.

Os trabalhos primários encontrados indicam diferentes sugestões de temas para trabalhos futuros apresentados pelos autores. São relatados, por exemplo, a realização de pesquisas com foco no conteúdo difundido no Moodle, a criação de um sistema para análise de discussão nos fóruns do Moodle, a mineração de dados no Moodle, a medição do desempenho dos alunos através das métricas de SNA, entre outros temas.

Os temas citados foram catalogados e classificados (vide Tabela 2). É importante mencionar que alguns trabalhos não apresentaram sugestões de pesquisas futuras, enquanto outros sugeriram mais de um tema.

\begin{tabular}{l|c}
\hline \multicolumn{1}{c|}{$\begin{array}{c}\text { Classificação de } \\
\text { Pesqisas Futuras }\end{array}$} & Porcentagem \\
\hline $\begin{array}{l}\text { Não apresenta trabalhos futu- } \\
\text { ros }\end{array}$ & $41 \%$ \\
\hline Aprimorar a própria pesquisa & $38 \%$ \\
\hline Estudos com foco no conteúdo & $3 \%$ \\
disseminado no Moodle & $3 \%$ \\
\hline Criação de um sistema para & \\
análise de discussão nos fó- \\
runs do Moodle \\
\hline $\begin{array}{l}\text { Desenvolvimento de ferramenta } \\
\text { que permita a visualização do }\end{array}$ \\
andamento do aluno no curso & $3 \%$ \\
\hline $\begin{array}{l}\text { Integração da ferramenta Face- } \\
\text { ted Browser ao Moodle }\end{array}$ & $3 \%$ \\
\hline Mineração de dados no Moodle & $3 \%$ \\
\hline Medir desempenho dos alunos \\
EaD através de métricas de snA
\end{tabular}

Tabela 2: Classificação de pesquisas futuras

\section{Conclusões}

A evolução dos AVAs no apoio ao processo de aprendizagem online e Blended Learning ocorre há pelo menos duas décadas e durante esses muitos anos a busca por técnicas que propiciasse e aumentasse a colaboração entre os professores, tutores e aprendizes consumiu grande parte dos trabalhos científicos nessa área. Mais recentemente, os olhos voltaram-se para o grande volume de dados gerados pelos AVAs e nas possibilidades de retor- 
no e de valor de informação que esses dados poderiam oferecer.

Compreender o comportamento dos atores envolvidos no processo de aprendizagem a partir da análise em tempo real das relações sociais existentes, intervindo nesse processo de forma a melhorar a experiência de aprendizagem, parece ser um objetivo desafiador especialmente considerando o número cada vez maior de cursos a distância e a dificuldade de acesso de grande parte da população mundial a uma educação de qualidade.

A quase totalidade dos trabalhos pesquisados são voltados à análise das interações de forma quantitativa, oferecendo um apoio aos professores e alunos de modo superficial. Uma vez quantificadas as interações, especialmente as sociais, sugere-se que sejam agregadas mecanismos de análise do discurso nas diferentes ferramentas colaborativas monitoradas do AVA.

O objetivo deste Mapeamento Sistemático foi o de fornecer uma visão geral de como a SNA tem contribuído com os estudos sobre E-Learning, bem como sugerir direcionamentos de estudo para pesquisas futuras neste área. $\mathrm{O}$ fato de identificarmos apenas 34 artigos científicos, ao final do estudo, demonstra que esta é uma subárea relativamente nova dentro da área de SNA e, consequentemente, promissora. Dado, também, o fato do aumento gradual da frequência de tais publicações na literatura (como evidencia a Figura 1) dos últimos anos.

Os estudos advindos deste Mapeamento evidenciam que a SNA pode contribuir de forma eficiente com a grande quantidade de dados presentes em ambientes de E-Learning, e que as possibilidades de representações gráficas associadas ao uso de métricas de SNA podem auxiliar os professores a entender as relações existentes nas comunidades online de alunos que se formam nas diferentes ferramentas colaborativas de um AVA.

Além disto, podemos mencionar outras contribuições alcançadas a partir deste estudo, a citar: compreensão mais precisa das interações que se formam em uma ambiente E-Learning; visão abrangente dos estudos publicados acerca desta temática; identificação dos principais focos e direcionamentos dados a pesquisa de SNA e E-learning; reconhecimento de novas possibilidades de pesquisa na área, entre outros.

Como trabalhos futuros, pretendemos aprofundar os estudos sobre a interação existente entre alunos que participam de fóruns de discussão em cursos à distância e averiguar por meio das métricas de Análise de Redes Sociais se este tipo de interação online por impactar, positivamente ou não, no desempenho do aluno em cursos desta modalidade, já que foi constatada a relevância desta temática. Bem como agregar a este Mapeamento Sistemático outras publicações científicas pesquisadas em veículos de publicação como anais de congressos, por exemplo, que versem sobre o conteúdo aqui apresentado, em complemento aos estudos encontrados.

\section{Referências}

[1] A. Brito, A. Duarte, J. Araújo, A. Torres, J. Cunha. Estudo da Utilização de Redes Sociais como Ferramenta de Avaliação de Participação. III Workshop de Avaliação. XXI Simpósio Brasileiro de Informática na Educação, SBIE. João Pessoa-PB. 2010.

[2] J. S. Carvalho. Redes e comunidades virtuais de aprendizagem: elementos para uma distinção. página196. Dissertação de Mestrado. Universidade de São Paulo, São Paulo. 2009.

[3] B. Kitchenham, D. Budgen, P. Brereton. Using mapping studies as the basis for further research-a participant-observer case study. Inform SoftwTechnol 53(6):638-651. 2011.

[4] A. Magalhães, G. Oliveira, S. Fialho. Indicadores em educação a distância: o uso da matriz de necessidades para ambientes virtuais de aprendizagem. XVII Congresso Internacional de Informática Educativa, TISE. Santiago, Chile. páginas 409-412, 2012.

[5] M. Marteleto. Análise de Redes Sociais - aplicações nos estudos de transferência da informação. Ci. Inf. Brasília, Brasil, PP 1-10. 2001.

[6] F. Medeiros, A. Gomes, R. Amorim, G. Medeiros. Redesigning Collaboration Tools to Enhance Social Presence in Online Learning Environments. In Collaboration and Technology. Springer Berlin Heidelberg. páginas 175191, 2013.

[7] R. Oliveira, J. Araújo, F. Medeiros, A. Brito. Monitoramento das Interações dos Aprendizes na Rede Social Twitter como Apoio ao Processo de Mediação Docente. Brazilian Workshop on Social Network Analysis and Mining, BrasNAM. XXXII Congresso da Sociedade Brasileira de Computação, CSBC. Curitiba, PR. 2012.

\section{Referências dos 34 artigos pesquisados}

[1] L. Ali, M. Asadi, D. Gaevi, J. Jovanovi, M. Hatala. Factors influencing beliefs for adoption of a learning analytics tool: an empirical study supplementary content. International Journal of Computers \& Education. páginas 130-148, 2013.

[2] N. Bijedic, I. Hamulic, E. Junuz, I. Maksumic, D. Radosav. Modeling sna result to improve learning community. Procedia - Social and Be- 
havioral Sciences. 2012.

[3] D. Cambridge, K. Perez-Lopez. First steps towards a social learning analytics for online communities of practice for educators. $L A K$ ' 12 : Proceedings of the 2nd International Conference on Learning Analytics and Knowledge. 2012.

[4] K. L. Cela, M. Á. Sicilia, S. Sánchez. Social Network Analysis in E-Learning Environments: A Preliminary Systematic Review. Educ Psychol Ver. 2014.

[5] E. Chamlee-Wright, J. A. Myers. Discovery and social learning in non-priced environments: an austrian view of social network theory. The Review of Austrian Economics. 2008.

[6] H. Cho, G. Gay, B. Davidson, A. Ingraffea. Social networks, communication styles, and learning performance in a cscl community. Computers \& Education. 2007.

[7] G. Cobo, D. Garca-Solrzano, J. A. Morn, E. Santamara, C. Monzo, J. Melenchn. Using agglomerative hierarchical clustering to model learner participation profiles in online discussion forums. $L A K$ '12: Proceedings of the 2nd International Conference on Learning Analytics and Knowledge. 2012.

[8] M. P. Cullar, M. Delgado, M. C. Pegalajar. Improving learning management through semantic web and social networks in e-learning environments. Expert Systems with Applications. 2011.

[9] A. Engel, C. Coll, A. Bustos. Distributed Teaching Presence and communicative patterns in asynchrouns learning: Name versus reply networks. International Journal Computer and Science. 2013.

[10] D. Garcia-Solórzano, G. Cobo, E. Santamaría, J. A. Morn, C. Monzo, J. Melenchn. Educational Monitoring Tool Based on Faceted Browsing and Data Portraits. $L A K$ '12: Proceedings of the 2nd International Conference on Learning Analytics and Knowledge. 2012.

[11] E. Gottardo, R. V. Noronha. Social networks applied to distance education courses: analysis of interaction in discussion forums. WebMedia '12: Proceedings of the 18th Brazilian symposium on Multimedia and the web. 2012.

[12] I. Hamulic, N. Bijedic. Social network analysis in virtual learning community at faculty of information technologies (fit), Mostar. Procedia Social and Behavioral Sciences. páginas 22692273, 2009.
[13] P. He. Evaluating students online discussion performance by using social network analysis. Information Technology: New Generations (ITNG) - Ninth International Conference on. 2012.

[14] H. Heo, K. Y. Lim, Y. Kim. Exploratory study on the patterns of online interaction and knowledge co-construction in project-based learning. International Journal of Computers \& Education. 2010.

[15] S. Hrastinski. What is online learner participation: a literature review. International Journal of Computers \& Education. páginas 1755-1765, 2008.

[16] F. Ke, C. Hoadley. Evaluating online learning communities. Educational Technology Research and Development. 2009.

[17] G. Lazakidou, S. Retalis. Using computer supported collaborative learning strategies for helping students acquire self-regulated problemsolving skills in mathematics. International Journal of Computers \& Education.2010.

[18] X. Liu, Q. He, Y. Tian, W. Lee, J. McPherson, J. Han. Event-based social networks: linking the online and offline social worlds. $K D D^{\prime}$ '12: Proceedings of the 18th ACM SIGKDD international conference on Knowledge discovery and data mining. 2012.

[19] A. B. F. Mansur, N. Yusof. Social learning network analysis model to identify learning patterns using ontology clustering techniques and meaningful learning. Computers \& Education. 2013.

[20] R. Mazza, V. Dimitrova. Coursevis: a graphical student monitoring tool for supporting instructors in web-based distance courses. International Journal of Human-Computer Studies. 2007.

[21] F. Medeiros. Architecture for Social Interactions Monitoring in Collaborative Learning Environments as a Support for the Teacher's Awareness. Advanced Learning Technologies (ICALT). IEEE 13th International Conference on. 2013.

[22] P. Mika. Social networks and the semantic web. WI '04: Proceedings of the 2004 IEEE/WIC/ACM International Conference on Web Intelligence. 2004.

[23] R. Rabbany, M. Takaffoli, O. R. Zaane. Social network analysis and mining to support the assessment of on-line student participation. SIGKDD Explorations Newsletter, Volume 13 Issue 2. 2012. 
[24] P. Reyes, P. Tchounikine. Mining learning groups activities in forum-type tools. $C S C L$ ' 05 : Proceedings of the 2005 conference on Computer support for collaborative learning. 2005.

[25] B. Rienties, Y. Hliot, D. Jindal-Snape. Understanding social learning relations of international students in a large classroom using social network analysis. Higher Education. 2013.

[26] B. Rienties, D. Tempelaar, P. V. Bossche, W. Gijselaers, M. Segers. The role of academic motivation in computer-supported collaborative learning. Computers in Human Behavior. páginas 1195-1206, 2009.

[27] H. Seridi, Y. Lafifi, N. Gouasmi. Clas: a collaborative learning awareness system. Procedia Social and Behavioral Sciences. páginas 480-485, 2012.

[28] A. Silva, A. Figueira. Depicting online interactions in learning communities. Global Engineering Education Conference (EDUCON). 2012.

[29] E. Sylvan. Who knows whom in a virtual learning network: applying social network analysis to communities of learners at the computer clubhouse. ICLS '06: Proceedings of the 7th international conference on Learning sciences. 2006.

[30] B. Tu, H. Wu, C. Hsieh, P. Chen. Establishing new friendships-from face-to-face to Facebook: a case study of college students. System Sciences (HICSS) - 44th Hawaii International Conference on. 2011.

[31] L. Wang. How social network position relates to knowledge building in online learning communities. Frontiers of Education in China. 2010.

[32] C. Yau, M. Straight, R. Bir, O. Addam, M. O. Shafiq, R. Alhajj. Examining Social Networks between Educational Institutions, Industrial Parterns, and the Candian Government. Information Reuse and Integration (IRI) - IEEE 13th International Conference on.2012.

[33] M. Zheng, H. Spires. Teachers interactions in an online graduate course on moodle: a social network analysis perspective. Meridian k-12 Computer Technologies Journal. 2011.

[34] L. Zhuhadar, R. Yang. Cyberlearners and learning resources. LAK '12: Proceedings of the 2nd International Conference on Learning Analytics and Knowledge. 2012. 